\title{
Phosphazene-catalyzed desymmetrization of cyclohexadienones by dithiane addition
}

\author{
Matthew A. Horwitz ${ }^{\ddagger}$, Elisabetta Massolo ${ }^{\ddagger}$ and Jeffrey S. Johnson*
}

\author{
Letter \\ Address: \\ Department of Chemistry, University of North Carolina at Chapel Hill, \\ Chapel Hill, NC 27599-3290, USA \\ Email: \\ Jeffrey S. Johnson* - jsj@unc.edu \\ * Corresponding author $\ddagger$ Equal contributors \\ Keywords: \\ conjugate addition; cyclohexadienones; dearomatization; \\ desymmetrization; dithiane; iminophosphoranes; organocatalysis; \\ phosphazenes
}

Beilstein J. Org. Chem. 2017, 13, 762-767.

doi:10.3762/bjoc. 13.75

Received: 21 January 2017

Accepted: 04 April 2017

Published: 24 April 2017

Associate Editor: T. P. Yoon

(c) 2017 Horwitz et al.; licensee Beilstein-Institut.

License and terms: see end of document.

\begin{abstract}
We report a desymmetrization of cyclohexadienones by intramolecular conjugate addition of a tethered dithiane nucleophile. Mild reaction conditions allow the formation of diversely functionalized fused bicyclic lactones. The products participate in facially selective additions from the convex surface, leading to allylic alcohol derivatives.
\end{abstract}

\section{Findings}

Desymmetrization has become a well-developed strategy for the construction of complex molecular frameworks [1-6]. Cyclohexadienones are multipurpose synthetic building blocks that have found a central role in desymmetrization methodologies. The functional groups present in these symmetrical molecules allow for a wide array of downstream transformations and they are all formed through a single reaction from cheap and readily available aromatic feedstocks [7-11]. These substrates have been successfully employed in a number of stereoselective desymmetrization reaction manifolds. Intramolecular Michael additions via enamine intermediates have been studied by the Gaunt [12] and Johnson groups [13]. The You group has disclosed methods for the intramolecular addition of amine [14] and bisphenylsulfonyl [15] nucleophiles using bifunctional cinchona alkaloid catalysts. The Sasai and Enders groups used a phosphinothiourea to enable a Rauhut-Currier reaction to form bicyclic enones [16]. The Tian and Lin group used alkyne-tethered cyclohexadienones in an arylrhodation/conjugate addition sequence that enantioselectively delivered oxabicyclo[4.3.0]nonanes [17]; the Lautens and Lan groups have also contributed to the further development of this reaction $[18,19]$. The Rovis group employed cyclohexadienone hydroperoxides in a chiral phosphoric acid-catalyzed $[1,2] /[1,4]$-addition cascade [20]. The same group also developed an acyl anion addition promoted by $N$-heterocyclic carbenes (NHC) that furnished bicyclic furanones via Stetter addition [21]; later, the 
You group developed an extension of this theme using the same catalytic manifold [22]. More recently, the Corey group has enabled the enantioselective conjugate reduction of prochiral cyclohexadienones using copper hydride generated in situ [23] Inspired by these advances, we sought to develop an alternative and complementary method invoking the dithiane moiety as an established and easily accessible glyoxylate anion surrogate [24-29]. This would in principle provide access to highly functionalized products with orthogonally protected carbonyl groups in a novel glycolic acid scaffold.

We envisioned utilizing para-quinol derivatives featuring a tethered nucleophile as desymmetrization substrates, with the intention of implementing a Brønsted base organocatalyzed addition (Scheme 1). This reaction would lead to bicyclic systems with the salient attribute of having a convex-concave facial differentiation, allowing subsequent diastereoselective transformations. With the aim of using a dithiane nucleophile, we selected 1,3-dithiane-2-carboxylic acid because of its relatively low $\mathrm{p} K_{\mathrm{a}}$ (compared with non-carboxylate substituted analogs) and the possibility of using an ester linkage as a tether. We found that the heretofore unknown dicyclohexylcarbodiimide (DCC) mediated coupling between para-quinols and 1,3dithiane-2-carboxylic acid proceeds in a straightforward manner in cases where $\mathrm{R}$ is unbranched (though it does work for $\mathrm{R}=\mathrm{Ph}$ ). Using this method, we were able to easily generate diversely functionalized dithiane-linked para-quinols to study the intramolecular cyclization.

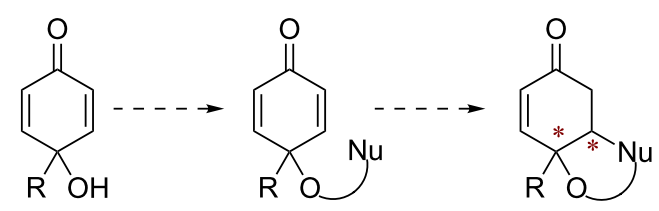

Scheme 1: Desymmetrization of cyclohexadienone by tethered nucleophile.
Based on a prior report [30] demonstrating the efficacy of phosphazene bases in deprotonating carboxylate dithianes, we selected the commercially available achiral superbase P2- $t$-Bu phosphazene to initiate the ring closure (Scheme 2) [31,32]. We found that in the simplest case, with the methyl-substituted para-quinol ester (1a), the reaction was complete in $30 \mathrm{~min}$ at ambient temperature with $20 \mathrm{~mol} \%$ catalyst [33]. Extending the length of the alkyl chain, the reaction proceeded similarly, even in the presence of a methyl ester or a TBS-protected primary alcohol (1b-d); a comparable result was observed with a phenyl substituent (1e). We considered that if a nucleophilic group were appended to the para-quinol, it would be possible to construct a 5-6-5 fused ring system. Indeed, when $\mathrm{R}=$ $\mathrm{CH}_{2} \mathrm{CH}_{2} \mathrm{NHBoc}(\mathbf{1 f})$, the desired tricyclic product $\mathbf{2 f}$ was obtained. In all cases only a single diastereomer was observed. In a substrate where a $\beta$-methyl group is present on the cyclohexadienone (1g), the reaction proved to be completely regioselective, only allowing conjugate addition to the less substituted position.

We attempted to render the reaction enantioselective using chiral iminophosphoranes (Figure 1) structurally related to P2-t-Bu phosphazene, which are known to be substantially more basic than trialkylamines [34]. With C1 [34-38] and C2 [39-55], we observed no product formation, presumably due to insufficient basicity. Though $\mathbf{C 3}[30,56,57]$ led to partial conversion of starting material, no appreciable enantioselectivity was observed.

To investigate the feasibility of a convex-facial addition, we subjected 2a to Luche reduction conditions (Scheme 3). We found this transformation to be completely diastereoselective, and an X-ray diffraction study [58] of the product confirmed our hypothesis regarding the facial selectivity, as the hydride was delivered to the convex face. An analogous reaction occurs when 2a is treated with $\mathrm{AlMe}_{3}$, affording the 1,2-addition product (Scheme 3) [59-63].<smiles>FC(F)(F)c1cc(NC(=S)NC(CN=Pc2ccccc2)c2ccccc2)cc(C(F)(F)F)c1</smiles>

C1

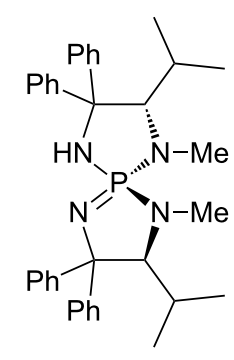

C2<smiles>CN1CCN2N=C3NCCN3C(c3ccccc3)C(c3ccccc3)NP3(=NC(c4ccccc4)[C@H](c4ccccc4)N4CCNC4=N3)N=C2N1</smiles>

C3 

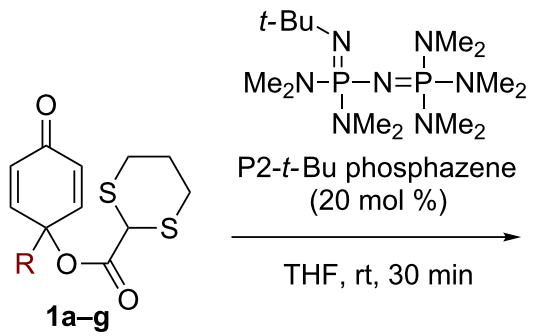

P2-t-Bu phosphazene

(20 $\mathrm{mol} \%)$

THF, rt, $30 \mathrm{~min}$

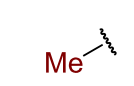

$\mathrm{Me} \xi^{\xi}$

$82 \%$ yield

$71 \%$ yield

2 b

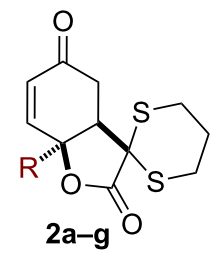

$2 a$<smiles>CCCO[Sb]</smiles><smiles>CCc1ccccc1</smiles>

$54 \%$ yield

2d

$81 \%$ yield

$2 \mathrm{e}$<smiles>O=C1C=CC(CCNC(=O)c2ccccc2)(OC(=O)C2SCCCS2)C=C1</smiles>

$1 f$

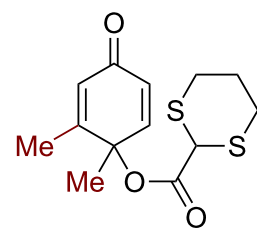

19

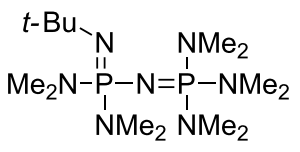

P2-t-Bu phosphazene $(20 \mathrm{~mol} \%)$

THF, rt, $30 \mathrm{~min}$ $\longrightarrow$ BocN"<smiles>CC(C)(C)OC(=O)N1CC2CC3(C(=O)O2)C2CC(=O)CC23SCCCS1</smiles>

$80 \%$ yield

$2 f$

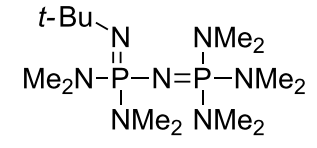

P2-t-Bu phosphazene (20 $\mathrm{mol} \%)$

THF, rt, $30 \mathrm{~min}$

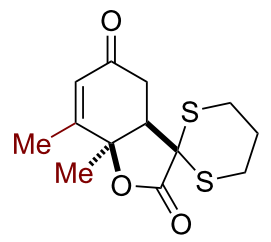

$73 \%$ yield

$2 \mathrm{~g}$

Scheme 2: Scope of the transformation.

We next sought to establish the glyoxylate anion equivalency of the dithiane substructure in our system. In order to reveal the masked carbonyl functionality, we rigorously applied reported dithiane deprotection conditions to 2a (Table 1). Despite extensive investigations, none of our efforts were fruitful, resulting in either no conversion, side reactions [64], or decomposition. We rationalized these disappointing results considering: 1) the crowded steric environment surrounding the dithiane moiety on the concave face of the bicycle, 2) the sensitive nature of this class of compounds, which stems from the highly reactive functional groups present, and 3) the strained character of the fivemembered $\alpha$-ketolactone (5) that would result from deprotection.
We attempted to synthesize $\mathbf{5}$ via an alternative route using $\mathrm{Cu}$ (II)-catalyzed aerobic oxidative deacylation [76] of the $\beta$-keto ester 6 (Scheme 4) [77]. The fact that this reaction also leads to decomposition of the starting material is cause for general concern about the feasibility of easily reaching the target substructure. In order to minimize the observed side reactions, we sought to apply the deprotection conditions to allylic alcohol 3. However, both the use of NBS and $\mathrm{HgCl}_{2} / \mathrm{HgO}$ were unsuccessful.

We further investigated the removal of the dithiane moiety via Raney nickel-promoted desulfurization (Scheme 5). To observe any substrate conversion, it was necessary to use a hydrogen at- 


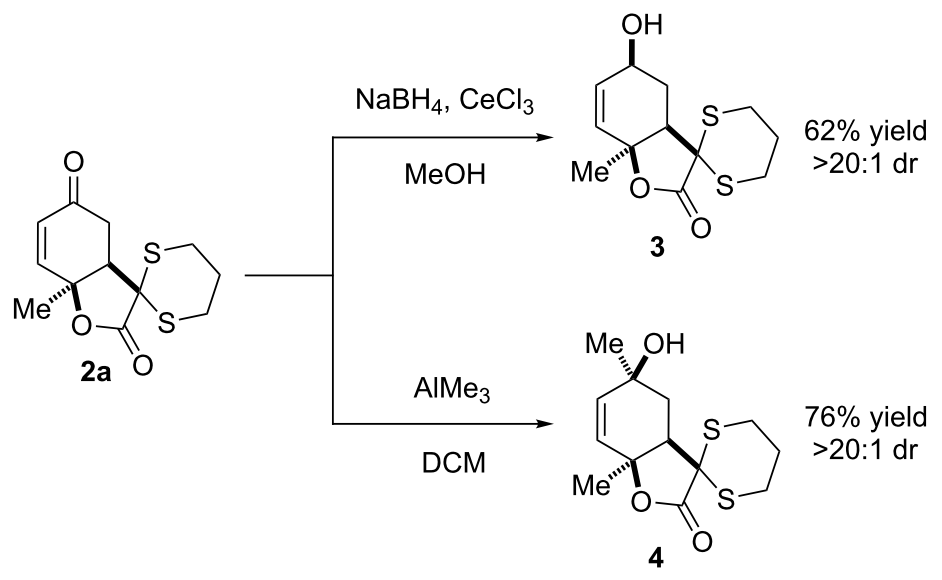

Scheme 3: Convex facial additions.

Table 1: Carbonyl deprotection conditions.<smiles>C[C@]12C=CC(=O)C[C@H]1C(=O)C(=O)O2</smiles>

\begin{tabular}{|c|c|c|}
\hline Entry & Conditions & Result \\
\hline $1[65]$ & $\mathrm{NBS}, \mathrm{MeCN} / \mathrm{H}_{2} \mathrm{O}, 0^{\circ} \mathrm{C}, 10 \mathrm{~min}^{\mathrm{a}}$ & side reaction \\
\hline $2[66]$ & $\mathrm{NBS}, \mathrm{AgNO}_{3}, \mathrm{MeCN} / \mathrm{H}_{2} \mathrm{O}, 0^{\circ} \mathrm{C}, 5 \mathrm{~min}^{\mathrm{b}}$ & side reaction \\
\hline $3[67]$ & $\mathrm{Phl}(\mathrm{OAc})_{2}, \mathrm{MeCN} / \mathrm{CH}_{2} \mathrm{Cl}_{2} / \mathrm{H}_{2} \mathrm{O}, 50^{\circ} \mathrm{C}, 18 \mathrm{~h}$ & no reaction ${ }^{c}$ \\
\hline $4[68]$ & $\mathrm{Hg}\left(\mathrm{ClO}_{4}\right)_{2}, \mathrm{MeOH} / \mathrm{CH}_{2} \mathrm{Cl}_{2}, \mathrm{rt}, 2 \mathrm{~h}$ & side reaction \\
\hline $5[69]$ & $\mathrm{HgCl}_{2}, \mathrm{HgO}, \mathrm{MeOH} / \mathrm{H}_{2} \mathrm{O}, 55^{\circ} \mathrm{C}, 18 \mathrm{~h}^{\mathrm{d}}$ & no reaction \\
\hline $6[70]$ & Mel, $\mathrm{MeCN} / \mathrm{H}_{2} \mathrm{O}$, reflux, $18 \mathrm{~h}$ & no reaction \\
\hline $7[71]$ & $m$-CPBA ${ }^{\mathrm{e}}, \mathrm{MeCN}, \mathrm{rt}, 18 \mathrm{~h}$, then $1 \mathrm{M} \mathrm{HCl}$, reflux, $4 \mathrm{~h}$ & decomposition \\
\hline $8[72]$ & $\mathrm{SbCl}_{5}, \mathrm{CH}_{2} \mathrm{Cl}_{2}, 0^{\circ} \mathrm{C}, 1 \mathrm{~h}$ & decomposition \\
\hline 9 [73] & $\mathrm{I}_{2}, \mathrm{NaHCO}_{3}$, acetone $/ \mathrm{H}_{2} \mathrm{O}, 50^{\circ} \mathrm{C}, 18 \mathrm{~h}$ & no reaction \\
\hline $10[74]$ & $\mathrm{CAN}^{\mathrm{f}}$, acetone $/ \mathrm{H}_{2} \mathrm{O}, 50^{\circ} \mathrm{C}, 18 \mathrm{~h}$ & no reaction \\
\hline $11[75]$ & Chloramine $\mathrm{T}, \mathrm{MeOH} / \mathrm{H}_{2} \mathrm{O}, 70^{\circ} \mathrm{C}, 18 \mathrm{~h}$ & no reaction \\
\hline
\end{tabular}

${ }^{a}$ Different solvent systems, such as acetone/ $\mathrm{H}_{2} \mathrm{O}$ and DMSO were used, stoichiometry was varied and the reaction was run also at rt and for longer times ( 4 and $18 \mathrm{~h}$ ) but in none of the cases was the desired product obtained. ${ }^{\mathrm{b}}$ The reaction was also run at rt for $18 \mathrm{~h}$, but the desired product was not obtained. 'Decomposition products were also observed. ${ }^{\mathrm{d}} \mathrm{The} \mathrm{MeCN} / \mathrm{H}_{2} \mathrm{O}$ solvent system was also used and the reaction was also run at rt and reflux, but in none of the cases was the desired product obtained. ${ }^{\mathrm{e}} m$-CPBA $=$ meta-chloroperbenzoic acid. ${ }^{\mathrm{f}} \mathrm{CAN}=$ ceric ammonium nitrate.<smiles>CC(=O)C1C(=O)O[C@]2(C)C=CC(=O)CC12</smiles>

6

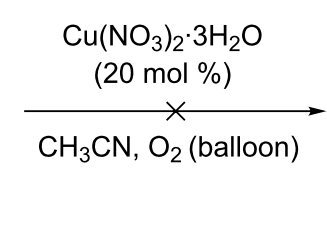

$\left(\mathrm{NO}_{3}\right)_{2} \cdot 3 \mathrm{H}_{2} \mathrm{O}$

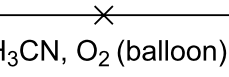

(n)<smiles>CC1(C)CCCC1C(=O)C(=O)O</smiles>

5

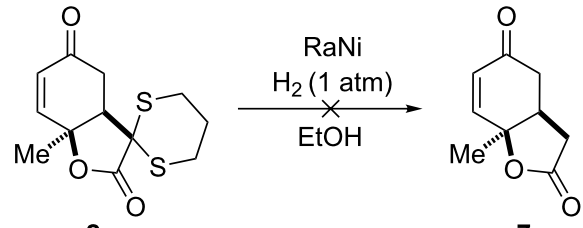

2a
7

Scheme 4: Attempted oxidative deacylation.

Scheme 5: Attempted desulfurization with Raney nickel. 
mosphere. Under those conditions, though the dithiane function was removed, degradation occurred.

\section{Conclusion}

In conclusion, we have developed a desymmetrizing intramolecular conjugate addition of a tethered dithiane moiety to paracresol-derived cyclohexadienones. The substrates are easily accessible from cheap starting materials and the reaction provides functionalized bicyclic lactones as a single diastereomer. The products of the reaction were able to undergo diastereoselective convex-facial additions. The carbonyl deprotection was unsuccessful and we hope that our efforts can serve as a cautionary tale for future synthetic planning involving related structures.

\section{Supporting Information}

\section{Supporting Information File 1}

Experimental procedures, characterization data and copies of ${ }^{1} \mathrm{H}$ and ${ }^{13} \mathrm{C}$ NMR spectra for final compounds. [http://www.beilstein-journals.org/bjoc/content/ supplementary/1860-5397-13-75-S1.pdf]

\section{Supporting Information File 2}

Crystallographic data.

[http://www.beilstein-journals.org/bjoc/content/ supplementary/1860-5397-13-75-S2.cif]

\section{Acknowledgements}

The project described was supported by Award R35 GM118055 from the National Institute of General Medical Sciences. X-ray crystallography was performed by Dr. Peter White.

\section{References}

1. Poss, C. S.; Schreiber, S. L. Acc. Chem. Res. 1994, 27, 9-17. doi:10.1021/ar00037a002

2. Magnuson, S. R. Tetrahedron 1995, 51, 2167-2213. doi:10.1016/0040-4020(94)01070-G

3. García-Urdiales, E.; Alfonso, I.; Gotor, V. Chem. Rev. 2005, 105, 313-354. doi:10.1021/cr040640a

4. Wang, M.; Feng, M.; Tang, B.; Jiang, X. Tetrahedron Lett. 2014, 55, 7147-7155. doi:10.1016/j.tetlet.2014.10.152

5. Borissov, A.; Davies, T. Q.; Ellis, S. R.; Fleming, T. A.; Richardson, M. S. W.; Dixon, D. J. Chem. Soc. Rev. 2016, 45, 5474-5540. doi:10.1039/C5CS00015G

6. Zeng, X.-P.; Cao, Z.-Y.; Wang, Y.-H.; Zhou, F.; Zhou, J. Chem. Rev. 2016, 116, 7330-7396. doi:10.1021/acs.chemrev.6b00094

7. Felpin, F.-X. Tetrahedron Lett. 2007, 48, 409-412. doi:10.1016/j.tetlet.2006.11.073

8. McKillop, A.; McLaren, L.; Taylor, R. J. K. J. Chem. Soc., Perkin Trans. 1 1994, 2047-2048. doi:10.1039/P19940002047
9. Jepsen, T. H.; Jensen, A. A.; Lund, M. H.; Glibstrup, E.; Kristensen, J. L. ACS Med. Chem. Lett. 2014, 5, 766-770. doi:10.1021/ml500094c

10. Canesi, S.; Bouchu, D.; Ciufolini, M. A. Org. Lett. 2005, 7, 175-177. doi:10.1021/ol048094v

11. Brown, P. D.; Willis, A. C.; Sherburn, M. S.; Lawrence, A. L. Org. Lett. 2012, 14, 4537-4539. doi:10.1021/ol302042u

12. Vo, N. T.; Pace, R. D. M.; O'Hara, F.; Gaunt, M. J. J. Am. Chem. Soc. 2008, 130, 404-405. doi:10.1021/ja077457u

13. Corbett, M. T.; Johnson, J. S. Chem. Sci. 2013, 4, 2828-2832. doi:10.1039/C3SC51022K

14. Gu, Q.; You, S.-L. Chem. Sci. 2011, 2, 1519-1522. doi:10.1039/C1SC00083G

15. Gu, Q.; You, S.-L. Org. Lett. 2011, 13, 5192-5195. doi:10.1021/ol202073p

16. Takizawa, S.; Nguyen, T. M.-N.; Grossman, A.; Enders, D.; Sasai, H. Angew. Chem., Int. Ed. 2012, 51, 5423-5426. doi:10.1002/anie.201201542

17. He, Z.-T.; Tian, B.; Fukui, Y.; Tong, X.; Tian, P.; Lin, G.-Q. Angew. Chem., Int. Ed. 2013, 52, 5314-5318. doi:10.1002/anie.201300137

18. Keilitz, J.; Newman, S. G.; Lautens, M. Org. Lett. 2013, 15, 1148-1151. doi:10.1021/ol400363f

19. Yu, Z.; Qi, X.; Li, Y.; Liu, S.; Lan, Y. Org. Chem. Front. 2016, 3, 209-216. doi:10.1039/C5Q000334B

20. Rubush, D. M.; Morges, M. A.; Rose, B. J.; Thamm, D. H.; Rovis, T. J. Am. Chem. Soc. 2012, 134, 13554-13557. doi:10.1021/ja3052427

21. Liu, Q.; Rovis, T. Org. Process Res. Dev. 2007, 11, 598-604. doi:10.1021/op600278f

22. Jia, M.-Q.; Liu, C.; You, S.-L. J. Org. Chem. 2012, 77, 10996-11001. doi:10.1021/jo3022555

23. Han, Y.; Breitler, S.; Zheng, S.-L.; Corey, E. J. Org. Lett. 2016, 18, 6172-6175. doi:10.1021/acs.orglett.6b03186

24. Eliel, E. L.; Hartmann, A. A. J. Org. Chem. 1972, 37, 505-506. doi:10.1021/jo00968a043

25. Takahashi, T.; Okano, T.; Harada, T.; Imamura, K.; Yamada, H. Synlett 1994, 121-122. doi:10.1055/s-1994-22762

26. Takahashi, T.; Tsukamoto, H.; Kurosaki, M.; Yamada, H. Synlett 1997, 1065-1066. doi:10.1055/s-1997-1549

27. Enders, D.; Bonten, M. H.; Raabe, G. Synlett 2007, 885-888. doi:10.1055/s-2007-970787

28. Enders, D.; Bonten, M. H.; Raabe, G. Angew. Chem., Int. Ed. 2007, 46, 2314-2316. doi:10.1002/anie.200604802

29. Liu, Q.; Perreault, S.; Rovis, T. J. Am. Chem. Soc. 2008, 130 , 14066-14067. doi:10.1021/ja805680z

30. Kondoh, A.; Oishi, M.; Takeda, T.; Terada, M. Angew. Chem., Int. Ed. 2015, 54, 15836-15839. doi:10.1002/anie.201508178

31. Massolo, E.; Benaglia, M.; Genoni, A.; Annunziata, R.; Celentano, G.; Gaggero, N. Org. Biomol. Chem. 2015, 13, 5591-5596. doi:10.1039/C5OB00492F

A significant increase in the dithiane carboxylate acidity is observed when the substrate is derivatized to an activated thioester.

32. Massolo, E.; Brenna, D.; Cozzi, F.; Raimondi, L.; Gaggero, N.; Benaglia, M. Synlett 2016, 27, 2716-2720. doi:10.1055/s-0036-1588306

33. Under the same reaction conditions, we observed that triethylamine was unable to promote the transformation and tetramethylguanidine provided only trace amounts of desired product after $13 \mathrm{~h}$.

34. Núñez, M. G.; Farley, A. J. M.; Dixon, D. J. J. Am. Chem. Soc. 2013, 135, 16348-16351. doi:10.1021/ja409121s 
35. Goldys, A. M.; Dixon, D. J. Macromolecules 2014, 47, 1277-1284. doi:10.1021/ma402258y

36. Farley, A. J. M.; Sandford, C.; Dixon, D. J. J. Am. Chem. Soc. 2015, 137, 15992-15995. doi:10.1021/jacs.5b10226

37. Robertson, G. P.; Farley, A. J. M.; Dixon, D. J. Synlett 2016, 27, 21-24. doi:10.1055/s-0035-1560530

38. Horwitz, M. A.; Zavesky, B. P.; Martinez-Alvarado, J. I.; Johnson, J. S. Org. Lett. 2016, 18, 36-39. doi:10.1021/acs.orglett.5b03127

39. Uraguchi, D.; Ueki, Y.; Ooi, T. J. Am. Chem. Soc. 2008, 130, 14088-14089. doi:10.1021/ja806311e

40. Uraguchi, D.; Ito, T.; Ooi, T. J. Am. Chem. Soc. 2009, 131, 3836-3837. doi:10.1021/ja810043d

41. Uraguchi, D.; Ueki, Y.; Ooi, T. Science 2009, 326, 120-123. doi:10.1126/science.1176758

42. Uraguchi, D.; Ito, T.; Nakamura, S.; Sakaki, S.; Ooi, T. Chem. Lett. 2009, 38, 1052-1053. doi:10.1246/cl.2009.1052

43. Uraguchi, D.; Ito, T.; Nakamura, S.; Ooi, T. Chem. Sci. 2010, 1, 488-490. doi:10.1039/CoSC00268B

44. Uraguchi, D.; Nakamura, S.; Ooi, T. Angew. Chem., Int. Ed. 2010, 49, 7562-7565. doi:10.1002/anie.201004072

45. Uraguchi, D.; Ueki, Y.; Ooi, T. Angew. Chem., Int. Ed. 2011, 50, 3681-3683. doi:10.1002/anie.201007752

46. Uraguchi, D.; Ueki, Y.; Ooi, T. Chem. Sci. 2012, 3, 842-845. doi:10.1039/C1SC00678A

47. Corbett, M. T.; Uraguchi, D.; Ooi, T.; Johnson, J. S. Angew. Chem., Int. Ed. 2012, 51, 4685-4689. doi:10.1002/anie.201200559

48. Uraguchi, D.; Yoshioka, K.; Ueki, Y.; Ooi, T. J. Am. Chem. Soc. 2012, 134, 19370-19373. doi:10.1021/ja310209g

49. Uraguchi, D.; Ueki, Y.; Sugiyama, A.; Ooi, T. Chem. Sci. 2013, 4, 1308-1311. doi:10.1039/C2SC22027J

50. Uraguchi, D.; Tsutsumi, R.; Ooi, T. J. Am. Chem. Soc. 2013, 135 8161-8164. doi:10.1021/ja403491j

51. Uraguchi, D.; Tsutsumi, R.; Ooi, T. Tetrahedron 2014, 70, 1691-1701. doi:10.1016/j.tet.2013.12.086

52. Tsutsumi, R.; Kim, S.; Uraguchi, D.; Ooi, T. Synthesis 2014, 46, 871-878. doi:10.1055/s-0033-1340818

53. Uraguchi, D.; Nakamura, S.; Sasaki, H.; Konakade, Y.; Ooi, T. Chem. Commun. 2014, 50, 3491-3493. doi:10.1039/C3CC49477B

54. Uraguchi, D.; Yamada, K.; Ooi, T. Angew. Chem., Int. Ed. 2015, 54, 9954-9957. doi:10.1002/anie.201503928

55. Horwitz, M. A.; Tanaka, N.; Yokosaka, T.; Uraguchi, D.; Johnson, J. S.; Ooi, T. Chem. Sci. 2015, 6, 6086-6090. doi:10.1039/C5SC02170G

56. Takeda, T.; Terada, M. J. Am. Chem. Soc. 2013, 135, 15306-15309. doi:10.1021/ja408296h

57. Takeda, T.; Kondoh, A.; Terada, M. Angew. Chem., Int. Ed. 2016, 55, 4734-4737. doi:10.1002/anie.201601352

58. CCDC1511800 contains the supplementary crystallographic data for this paper. These data can be obtained free of charge from the Cambridge Crystallographic Centre via http://www.ccdc.cam.ac.uk/data_request/cif.

59. Carreño, M. C.; González, M. P.; Ribagorda, M. J. Org. Chem. 1996, 61, 6758-6759. doi:10.1021/jo961126a

60. Carreño, M. C.; González, M. P.; Ribagorda, M. J. Org. Chem. 1998, 63, 3687-3693. doi:10.1021/jo980084i

61. Carreño, M. C.; Ribagorda, M.; Somoza, Á.; Urbano, A. Angew. Chem., Int. Ed. 2002, 41, 2755-2757. doi:10.1002/1521-3773(20020802)41:15<2755::AID-ANIE2755>3.0.CO ;2-A
62. Carreño, M. C.; Merino, E.; Ribagorda, M.; Somoza, Á.; Urbano, A. Org. Lett. 2005, 7, 1419-1422. doi:10.1021/ol050287a

63. Carreño, M. C.; Merino, E.; Ribagorda, M.; Somoza, Á.; Urbano, A. Chem. - Eur. J. 2007, 13, 1064-1077. doi:10.1002/chem.200601330

64. When NBS was used, the unpurified ${ }^{1} \mathrm{H}$ NMR spectrum was suggestive of bromination at the ketone $\alpha$-position, although the product was not isolable by column chromatography. With $\mathrm{Hg}\left(\mathrm{ClO}_{4}\right)_{2}$, an unidentified rearomatized product was observed.

65. Corey, E. J.; Erickson, B. W. J. Org. Chem. 1971, 36, 3553-3560. doi:10.1021/jo00822a019

66. Burks, E. A.; Johnson, W. H., Jr.; Whitman, C. P. J. Am. Chem. Soc 1998, 120, 7665-7675. doi:10.1021/ja9808402

67. Gao, A. X.; Hamada, T.; Snyder, S. A. Angew. Chem., Int. Ed. 2016, 55, 10301-10306. doi:10.1002/anie.201604744

68. Tsuboi, K.; Ichikawa, Y.; Jiang, Y.; Naganawa, A.; Isobe, M. Tetrahedron 1997, 53, 5123-5142. doi:10.1016/S0040-4020(97)00230-5

69. Nicewicz, D. A.; Yates, C. M.; Johnson, J. S. Angew. Chem., Int. Ed. 2004, 43, 2652-2655. doi:10.1002/anie.200353354

70. Fetizon, M.; Jurion, M. J. Chem. Soc., Chem. Commun. 1972 , 382-383. doi:10.1039/C39720000382

71. Smith, A. B.; Dorsey, B. D.; Visnick, M.; Maeda, T.; Malamas, M. S. J. Am. Chem. Soc. 1986, 108, 3110-3112. doi:10.1021/ja00271a054

72. Kamata, M.; Otogawa, H.; Hasegawa, E. Tetrahedron Lett. 1991, 32, 7421-7424. doi:10.1016/0040-4039(91)80123-N

73. Nicolaou, K. C.; Bunnage, M. E.; McGarry, D. G.; Shi, S.; Somers, P. K.; Wallace, P. A.; Chu, X.-J.; Agrios, K. A.; Gunzner, J. L.; Yang, Z. Chem. - Eur. J. 1999, 5, 599-617. doi:10.1002/(SICI)1521-3765(19990201)5:2<599::AID-CHEM599>3.0. $\mathrm{CO} ; 2-\mathrm{N}$

74. La Claire, J. J.; Lansbury, P. T.; Zhi, B.-x.; Hoogsteen, K. J. Org. Chem. 1995, 60, 4822-4833. doi:10.1021/jo00120a027

75. Emerson, D. W.; Wynberg, H. Tetrahedron Lett. 1971, 12, 3445-3448. doi:10.1016/S0040-4039(01)97201-6

76. Steward, K. M.; Johnson, J. S. Org. Lett. 2011, 13, 2426-2429. doi:10.1021/ol200649u

77. Tello-Aburto, R.; Kalstabakken, K. A.; Volp, K. A.; Harned, A. M. Org. Biomol. Chem. 2011, 9, 7849-7859. doi:10.1039/C1OB06125A

\section{License and Terms}

This is an Open Access article under the terms of the Creative Commons Attribution License (http://creativecommons.org/licenses/by/4.0), which permits unrestricted use, distribution, and reproduction in any medium, provided the original work is properly cited.

The license is subject to the Beilstein Journal of Organic Chemistry terms and conditions: (http://www.beilstein-journals.org/bjoc)

The definitive version of this article is the electronic one which can be found at: doi: $10.3762 /$ bjoc. 13.75 